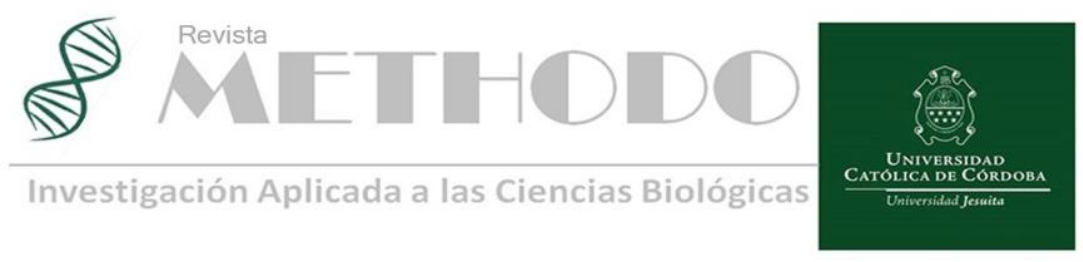

ARTICULO DE REVISION Rev. Methodo 2020;5(1):30-34 https://doi.org/10.22529/me.2020.5(1)08

Recibido 23 Oct. 2019 | Aceptado 16 Dic. 2019 |Publicado 31 Mar. 2020

\title{
Las actividades profesionales confiables: una herramienta para hacer operativo el concepto de competencia
}

\section{Reliable professional activities: a tool to make the concept of competition operational}

\author{
Marcelo García Diéguez ${ }^{1}$, Roberta Inés Ladenheim² \\ 1.Universidad Nacional del Sur. Departamento de Ciencias de la Salud \\ 2.Instituto Universitario Hospital Italiano \\ Correspondencia: Marcelo García Diéguez; e-mail: gdieguezm@gmail.com.
}

\section{Resumen}

La educación de profesionales de la salud ha evolucionado de un enfoque con énfasis en la adquisición de contenidos a uno vinculado al alcance de resultados predeterminados, constituidos por el desarrollo de competencias por parte de los alumnos. En este contexto, surge el concepto de Actividades profesionales confiables (APROC) como una manera de vincular las competencias a la práctica profesional cotidiana y ofrecer una herramienta que permite operativizarlas facilitando la planificación de la enseñanza. El desarrollo gradual de la competencia implica que los alumnos requieren supervisión decreciente, constituyéndose la evaluación de la práctica en una herramienta fundamental para decidir qué tan apto se encuentra un estudiante para llevar adelante una tarea determinada en un contexto concreto y con qué grado de supervisión. Las APROCs ofrecen un modelo concreto de niveles de supervisión que permiten acompañar a los alumnos en el desarrollo de su autonomía minimizando los riesgos para los pacientes.

Palabras clave: Educación basada en competencias, Actividades profesionales confiables, supervisión, Evaluación basada en el trabajo.

\begin{abstract}
Health professionals education has evolved from an approach with emphasis on the acquisition of content to an outcome-based one, characterized by the development of competencies. In this context, the concept of Entrustable Professional Activities (EPAs) emerges as a way of linking competencies to practice and operationalizing them in a way that facilitates educational planning. Gradual development of competency implies that students will need decreasing supervision, practice assessment thus becoming a key tool to determine if one trainee can execute a task in a specific context and with what level of supervision. EPAs offer a concrete scale of supervision to accompany trainees in the development of competence and autonomy minimizing the risk and guaranteeing patient safety.
\end{abstract}

Keywords: Competency, based medical education. Entrustable professional activities, supervisión, work, based assessment.

Revista Methodo: Investigación Aplicada a las Ciencias Biológicas. Universidad Católica de Córdoba Jacinto Ríos 571 Bo Gral. Paz. X5004FXS. Córdoba. Argentina. Tel.: (54) 3514517299 / Correo: methodo@ucc.edu.ar / Web: methodo.ucc.edu.ar | ARTICULO DE REVISION Rev. Methodo 2020;5 (1):30-34 


\section{Introducción}

Si buscamos a lo largo de la historia de la educación médica hay hitos que perduran en el tiempo. El primero de estos es el paradigmático informe Flexner ${ }^{1}$ que marcó la educación médica por casi un siglo, con un énfasis marcado en la importancia de los contenidos. A partir de la mitad del siglo XX comienzan a generar preocupación las metodologías de enseñanza-aprendizaje, movilizados por la aparición de nuevas teorías educacionales. A 100 años de aquel informe las Recomendaciones del Reporte de la Carnegie $2010^{2}$ introducen un nuevo aspecto, sugieren que la educación médica actual debe basarse en estándares fijos y caminos flexibles. Deja en claro que es necesaria la integración de conocimiento y experiencia clínica. Señala dicho reporte, que deben promoverse los hábitos de investigación e innovación y promover la formación de una identidad profesional. Todo ello lleva a pensar que el resultado de la formación es una construcción compleja, que denominamos competencia. El problema con el concepto de competencia no es sólo comprenderla, sino definirla y operativizarla para hacerla asequible a docentes y estudiantes. La dificultad de la Educación Basada en Competencias (EBC), es su implementación. Resulta interesante como Hodges desnuda este problema mostrando como en el uso de la palabra subyace una serie de discursos que van desde aquel casi flexneriano de entenderla como el acopio de conocimiento, a los que se quedan sólo en los aspectos de su evaluación. Incluso detecta en su estudio el erróneo discurso de pensar que la educación basada en resultados se restringe a la visión mecanicista de la producción ${ }^{3}$.

Para avanzar, aceptemos una de las definiciones más extendidas en la literatura, que señala que la competencia profesional es "la capacidad de un profesional de utilizar su buen juicio, así como también los conocimientos, habilidades, y actitudes asociadas a la profesión para solucionar los problemas complejos que se presentan en el campo de su actividad profesional". Esta definición destaca varios aspectos claves, por un lado, la multiplicidad de recursos necesarios, su clara dependencia contextual, y por el otro que sólo son evidenciadas en las acciones ${ }^{4}$.

El concepto de competencia va íntimamente entrelazado al de educación basada en resultados cuyos aspectos claves son

- Énfasis en Resultados no en el proceso: la clave es el logro, no sólo lo que se hace

- Focalizada en capacidades: que integran conocimiento, habilidades, y actitudes
- Independiente del tiempo: la duración del entrenamiento debe adaptarse a las diferencias individuales

- Individualizada: los aprendices y los contextos no son siempre iguales.

En la aplicación cotidiana de la EBC, muchas veces las competencias son confundidas con cosas que no lo son, como ser colecciones de largas listas con enunciados que pretenden reflejar lo que se ha aprendido o lo que debe aprenderse, una lista de actividades educativas, o listas de cotejo de rendimiento. Es frecuente ver, al inicio de los programas, una lista de competencias sin un plan para adquirirlas o evaluarlas, sin que se dé una explicación de cómo se relacionan con otros aspectos de un plan curricular como los contenidos o las actividades educacionales. Los críticos señalan que las competencias traen un concepto reduccionista y utilitario de la educación, que pierde de vista los procesos cognitivos detrás del desarrollo de ella ${ }^{78}$

Pero para una aplicación juiciosa de este modelo debe entenderse que focalizar en resultados es saber que el estudiante (o residente) demuestra competencias (y esto se registra) que lo ponen en condiciones de estar listo para proseguir a la siguiente etapa de su formación Por ello requiere evaluaciones que determinen que estas cosas se hacen consistentemente y dentro de las necesidades contextuales del entorno clínico. Su implementación pone de relieve la necesidad de centrarse en el estudiante, en el desarrollo de sus habilidades y en los diferentes tiempos que cada individuo tiene para lograr ese desarrollo. Para poder seguir ese progreso es necesario comenzar a identificar hitos claramente definidos ${ }^{5}$.

Diversas instituciones han tomado el camino de definir las dimensiones que componen la competencia del médico ya sea usando un modelo de áreas de competencia como ACGME o un modelo de roles del profesional como el modelo canadiense de CanMed ${ }^{10}$.

Aún así el aspecto más complejo para los educadores es traducir estos conceptos a programas educativo que contribuyan de la manera más coherente a su desarrollo, buscando el camino para corto y adecuado para ese cometido.

\section{Las actividades profesionales confiables}

Fueron descriptas por Olle Ten cate hace 15 años como una forma de acercar las teorías de la competencia a la práctica. Se definen como una parte del trabajo profesional esencial en un contexto dado, ejecutable en forma independiente 
y dentro de un tiempo determinado, observable y medible en el proceso y los resultados. Es decir que se trata de una unidad de la práctica que puede ser confiada a un profesional en entrenamiento una vez que alcanzó un grado suficiente de competencia.

El concepto engloba tres aspectos

- Actividades: Tareas que deben ser hechas, deben estar listadas en las especificaciones laborales.

- Profesionales: porque son confinadas a profesiones con regulación legal u alto nivel de calificación.

- "Confiables" que surge del término en inglés "Entrustable" que resulta de difícil traducción al español. (Confiable): acto que requiere confianza - de los colegas, los pacientes, la sociedad. Prohibido para personas no calificadas.

Para que una actividad constituya una APROC debe tratarse de una parte esencial del trabajo profesional y no simplemente una habilidad médica, debe requerir conocimientos, habilidades y actitudes determinadas, constituir un rendimiento reconocible que es exclusivo de la práctica médica y de determinada especialidad, debe ser ejecutable en un período de tiempo, observable y con un resultado medible ${ }^{12}$.

Al referirnos a las competencias hablamos de descripciones personales mientras que las APROCs describen tareas profesionales. De esta manera, las APROCs son un concepto que facilita APROCs llevan las competencias a la práctica diaria. Por ejemplo, una APROC de egreso de carrera de medicina puede ser "Recoger una historia clínica y realizar el examen físico", mientras que una APROC de una especialidad médica como la medicina de familia puede ser "manejo del paciente con dolor torácico". Para lograr las APROCs se requiere poner en juego múltiples dimensiones de las competencias. Si tomamos el ejemplo de una APROC como "realizar asesoramiento telefónico" esta requiere para su realización que el residente integre competencias de más de un dominio, o si usamos un modelo de roles, tratándose de una tarea compleja requiere el desempeño utilizando más de un rol en simultáneo.

El segundo aspecto clave de las APROCs es que definen el grado de autonomía con que estas actividades pueden ser realizadas. Esto vincula directamente el concepto de competencia con el de evaluación, y resalta el rol de la supervisión en el seguimiento del desarrollo de las APROCs. De esta manera, se redefine el ser competente, "Más allá del límite de habilidad que permite confiar en sus acciones sin supervisión". La competencia se alcanza cuando confiamos en el alumno/ residente puede realizar una determinada actividad profesional, con determinado nivel, que permite la práctica no supervisada y confianza completa. Otro aspecto derivado de este concepto es que un mismo residente desarrollo de las APROCs en diversos momentos, a partir de la práctica supervisada, con desarrollo gradual

Definir cuáles son las APROCs que caracterizan determinada profesión o especialidad es un proceso de consenso entre actores. Se describieron una serie de pasos necesarios para su correcto diseño Tabla 1. Una vez construido el conjunto de APROC se recomienda hacer una verificación que permita asegurar que estas responden al sentido central de la profesión o especialidad que pretender representar. Para ello recientemente Taylor ha desarrollado una rúbrica que permite hacerlo en forma válida y confiable ${ }^{16}$.

Tabla 1: Pasos en el desarrollo de las APROCs.

\begin{tabular}{l}
$\begin{array}{l}\text { Fase 1: identificar los dominios. Como fuentes se utilizan la } \\
\text { opinión de profesionales experimentados, opinión de } \\
\text { aprendices, grupos focales, opinión de docentes, observación. } \\
\text { El producto de esta fase consiste en un mapa de APROCs. }\end{array}$ \\
\hline $\begin{array}{l}\text { Fase 2: Mapeo y confirmación, consiste en el chequeo de que } \\
\text { todos los aspectos están cubiertos. Se realiza mediante } \\
\text { consulta de expertos. }\end{array}$ \\
\hline $\begin{array}{l}\text { Fase 3: Descripción. Redacción más detallada de cada una de } \\
\text { las APROC. Se realiza mediante consulta de expertos. Delphi. El } \\
\text { Producto de esta fase es la descripción de las APROCs. }\end{array}$ \\
\hline $\begin{array}{l}\text { Fase 4: Confirmación del contenido. Revisión de la descripción. } \\
\text { Determinación de los elementos de verificación. Talleres con } \\
\text { expertos. }\end{array}$ \\
\hline Fase 5: Finalización. Redacción final y verificación
\end{tabular}

\section{La evaluación en el contexto de las aprocs}

En el contexto de las APROCs, la evaluación se considera una herramienta para definir si un estudiante (o residente) se encuentra en condiciones de realizar una actividad determinada sin supervisión. Dependiendo de cuál es la tarea y su grado de desarrollo de experticia en la misma, un estudiante o residente puede requerir diversos niveles de supervisión. En el nivel uno, solo puede ser un observador, dado que el conocimiento y las destrezas de las que dispone no son suficientes para el desempeño, es decir que se necesita la supervisión total. En un segundo nivel, es suficiente la supervisión limitada, que se realiza en forma directa en caso de que se requiera la intervención en el momento del supervisor. En un tercer nivel es posible la práctica no supervisada, es decir, con supervisión "desde la trastienda". Finalmente, cuando el estudiante alcanza cierto

Revista Methodo: Investigación Aplicada a las Ciencias Biológicas. Universidad Católica de Córdoba. 
grado de competencia podemos decir que se encuentra en condiciones de impartir la supervisión a otra persona en formación Tabla $2^{17}$. Decidir qué nivel de supervisión requiere cada estudiante para cada actividad, o la decisión de que un estudiante puede llevar adelante una tarea sin supervisión (decisión confiable justificada) implica una decisión activa por parte de un docente supervisor, que debe tener datos confiables para tomarla y con criterios claros y comunes a todos los supervisores.

Tabla 2. Niveles de Supervisión según el grado de supervisión que requiera el estudiante o residente para realizar determinada APROC.

\begin{tabular}{|l|l|}
\hline 1 & $\begin{array}{l}\text { No está en condiciones de realizar la APROC. Posee conocimientos o } \\
\text { habilidades que le permiten observar la actividad }\end{array}$ \\
\hline 2 & Puede realizar la APROC bajo supervisión directa en el lugar \\
\hline 3 & Puede realizar la APROC bajo supervisión indirecta, disponible en minutos \\
\hline 4 & Puede actuar sin supervisión, bajo control distante por parte del supervisor \\
\hline 5 & Puede proveer supervisión a otros eestudiantes o residentes. \\
\hline
\end{tabular}

La planificación de las actividades educativas requiere conocer en qué nivel de desarrollo de cada APROC se encuentra cada estudiante, de manera de poder facilitar el aprendizaje, pero también para garantizar la seguridad en la atención de los pacientes. En el ámbito de la enseñanza clínica, los supervisores de manera cotidiana permiten que sus estudiantes (o residentes) lleven adelante tareas, no siempre con la certeza de que están "aptos" para hacerla. Es clave que dispongan de información producto de la evaluación del desempeño para la toma de estas decisiones. Esto pone de relieve que la información más relevante es la que se recoge de la práctica, y por ende es importante conocer las limitaciones y sesgos de la evaluación basada en el trabajo. Este tipo de evaluación gana en validez, pero las limitaciones vinculadas a su confiabilidad obligan a aumentar el número de evaluaciones y combinar diversas fuentes de información. Simultáneamente, la evaluación en el contexto de las APROCs lleva a pensar una evolución de la evaluación que pasa de ser una evaluación del aprendizaje, un registro de progreso, a un modelo conceptual en el que los procesos de evaluación estén función del aprendizaje, son para el aprendizaje. Este cambio conceptual es lo que hoy se conoce como evaluación programática, un modelo en él se ha ampliado tanto el tipo y naturaleza de la información que se recolecta, como así el uso que se le da. En estos modelos se prioriza la decisión colectiva al valor psicométrico individual de las medidas ${ }^{18}$

La transferencia de confianza describe un punto en el que el supervisor autoriza a un estudiante/residente a realizar una tarea de manera independiente. Esta decisión de confianza incluye más que la simple evaluación de la habilidad del estudiante o residente. La decisión de transferir responsabilidad a un aprendiz debería ocurrir cuando se juzga que tiene la preparación suficiente, cuando su habilidad corresponde con la complejidad de la situación del paciente y los riesgos son aceptables, entendiendo que cualquier decisión de delegación implica asumir un riesgo ${ }^{19}$ 20.

Los factores que influyen en que confiemos en que un estudiante podrá llevar adelante una tarea sin supervisión incluyen cuatro puntos fundamentales. La "confianza" requiere, por un lado, que el docente tenga la certeza de que el estudiante posee la habilidad, es competente en el sentido tradicional. Pero esta habilidad debe estar acompañada por integridad, es decir honestidad y benevolencia; Confiabilidad entendida como un comportamiento consciente y consistente; y finalmente humildad, el discernimiento de sus limitaciones y la predisposición a pedir ayuda en caso de necesitarla.

En la formación en servicio, el alumno/residente se forma para ser confiable y debe volverse confiable; pero sabemos que es habitual que los graduados estén habilitados a llevar adelante actividades en las que los supervisores nunca los han observado, e incluso que nunca han tenido la oportunidad de realizar. En este sentido, las decisiones de transferencia de confianza requieren una estimación de la competencia adaptativa. Estas decisiones ocurren diariamente de una forma implícita. Es necesario pasar de criterios implícitos a criterios explícitos para tomar esta decisión, cuando son implícitos refieren a criterios propios o institucionales, criterios referidos a la norma, criterios referidos al proceso o progreso del propio aprendiz; la decisión se toma sin tener en cuenta si pueden o no llevar a cabo la actividad. Las APROCs promueven que la delegación de responsabilidad se decida por unidades separadas de práctica profesional, posibilitando la participación gradual y legítima de los sujetos en formación, transformando la evaluación tradicional en decisiones de delegación como marco de referencia.

Las APROCs vinculan el concepto de competencia con la práctica profesional, facilitando su aplicación para la planificación y el monitoreo del desarrollo de la misma. Ofrecen, además un modelo de supervisión escalonado que se ajusta al nivel de alcance de la competencia de cada estudiante en cada tarea, requiriendo una evaluación de la práctica que sea insumo para el aprendizaje. De esta manera, se constituyen en un camino para la implementación de la educación basada en competencias, a través de conceptos de fácil compresión para todos docentes del ámbito clínico ya que reflejan su práctica cotidiana.

\section{Bibliografía}

1. Flexner A. Medical Education in the United States and Canada. Washington, DC: Science and Health Publications, Inc.; 1910 
2. Cooke M, Irby DM, O’Brien MC. Educating Physicians: A call for reform of medical school and residency. 1st Ed, San Francisco: Jossey-Bass, 2010

3. Hodges B. Medical education and the maintenance of incompetence. Med Teach 2006; 28:690-6

4. Fernandez N, Dory V, Ste-Marie LG, Chaput M, Charlin B, Boucher A. Varying conceptions of competence: an analysis of how health sciences educators define competence. Med Educ 2012; 46:357-365

5. Frank JR, Snell LS, Cate OT, Holmboe ES, Carraccio C, Swing SR, Harris P, Glasgow NJ, Campbell C, Dath D, Harden RM, Iobst W, Long DM, Mungroo R, Richardson DL, Sherbino J, Silver I, Taber S, Talbot M, Harris KA. Competency-based medical education: theory to practice. Med Teach. 2010; 32:63845.

6. Albanese M, Mejicano G, Mullan P, Kokotailo P, Gruppen L. Defining characteristics of educational competencies. Med Educ 2008; 42:248-55

7. Talbot M. Monkey see, monkey do: a critique of the competency model in graduate medical education. Med Educ. 2004; 38:587-92.

8. Grant $\mathrm{J}$ The incapacitating effects of competence: A critique. Health Sci Educ Theory Pract 1999; 4:271-277.

9. Andolsek K, MedstarJP, Hauer KE, Edgar L, Holmboe E. Clinical Competency Committee Guidebook: a guidebook for programs. 2nd ed. Chicago: Acreditation Council for Graduate Medical Education: 2017. Disponible en: https://www.acgme.org/Portals/0/ACGMECl inicalCompetencyCommitteeGuidebook.pdf. Acceso 6 dic 2019

10. Frank JR, Snell L, Sherbino J, editors. Can Meds 2015 Physician Competency Framework. Ottawa: Royal College of Physicians and Surgeons of Canada; 2015.

11. Ten Cate O. Entrustability of professional activities and competency-based training. Med Educ 2005; 39:1176-7.

12. Ten Cate O, Scheele F. Competency-based postgraduate training: Can we bridge the gap between theory and clinical practice? Acad Med 2007; 82 (6): 542-7.

13. Obeso V, Brown D, Aiyer M, Barron B, Bull J, Carter T, Emery M, Gillespie C, Hormann M, Hyderi A, Lupi C, Schwartz M, Uthman M, Vasilevskis EE, Yingling S, Phillipi C, eds.; for Core EPAs for Entering Residency Pilot Program. Toolkits for the 13 Core Entrustable Professional Activities for Entering Residency. Washington, DC: Association of American Medical Colleges; 2017. Disponible en aamc.org/initiatives/coreepas/publicationsand presentations. Acceso 6 dic 2019

14. Shaughnessy A, Sparks J, Cohen-Osher M, Goodell K, Sawin G, Gravel J. Entrustable Professional Activities in Family Medicine. J Grad Med Ed 2013; 5:112-118.

15. Chen HC, van den Broek WE, ten Cate O. The case for use of entrustable professional activities in undergraduate medical education. Acad Med 2015; 90:431-436.

16. Taylor DR, Park YS, Egan R, Chan MK, Karpinski J, Touchie C, Snell LS, Tekian A. EQual, a novel rubric to evaluate entrustable professional activities for quality and structure. Acad Med. 2017;92: S110-S117

17. Ten Cate O. Formación médica y evaluación basadas en las competencias: ¿de qué se trata y cómo puede relacionarse con la realidad de la práctica clínica?Rev Arg Cardiol 2011; 79:405- 408

18. Schuwirth LW, Van der Vleuten CP. Programmatic assessment: from assessment of learning to assessment for learning.Med Teach. 2011; 33:478-85

19. Ten Cate O. Entrustment as assessment: recognizing the ability, the right, and the duty to act. J Grad Med Edu 2016, 8: 261-262

20. Ten Cate O, Hart D, Ankel F, Busari J, Englander R, Glasgow N, et al. Entrustment decision-making in clinical training. Acad Med. 2016; 91:191-198

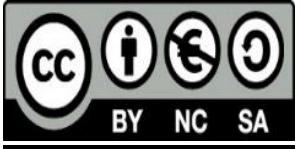

